\title{
Proximal femoral derotation osteotomy in children with CP : long term outcome and the role of age at time of surgery
}

\author{
Lieven Vermuyten, Katleen Desloovere, Guy Molenaers, Anja Van Campenhout
}

UZ Leuven Department of Orthopaedic surgery, Leuven, Belgium

The femoral derotation osteotomy (FDO) is seen as the golden standard treatment in children with cerebral palsy and internal rotated gait. This study provides quantitative evidence in support of the beneficial effect of FDO after long term follow up.

Retrospective clinical and kinematic evaluation of 31 CP patients (55 operated limbs) pre-, 1 and 3 years postoperatively after proximal FDO was conducted for a minimal follow-up of 3 years. This group consisted of 20 men and 11 women, aged $10.68 \pm 3.31$ years at the time of surgery. Minimum follow up was 3 years (3.16 \pm 0.53 years), with 22 patients (38 operated limbs) having an additional follow up at 5 years $\mathbf{( 5 . 0 2 \pm 0 . 4 9}$ years). Age at FU3 and FU5 was 14.06 \pm 3.52 years and $15.39 \pm 3.08$ years respectively.

A set of clinical and kinematic parameters were analyzed and showed a significant correction of mean hip rotation and femoral anteversion after FDO. Further plotting of individual data comparing 3 or 5 year postoperative values to 1 year postoperative values showed no further significant changes, indicating sustained correction of internally rotted gait until end of our follow up. Plotting mean hip rotation in stance as well as kinematic knee parameters according to age grouped cohorts could not show age at time of surgery to be a significant factor in recurrence of internally rotated gait or preoperative disturbances of knee motion in the sagittal plane.

This study provides quantitative evidence on the beneficial effect of FDO, a surgical technique to improve internally rotated gait in cerebral palsy patients with spastic diplegia.

Pre- and postoperative clinical and kinematic parameters are compared and results are discussed.
Minimum follow up was 3 years with a mean follow up of $4.65 \pm 0.83$ years. The effect of age at time of surgery on recurrence and kinematic parameters were studied.

Keywords : cerebral palsy ; femoral derotation osteotomy ; gait analysis ; age.

\section{INTRODUCTION}

Cerebral Palsy (CP) is the most common cause of chronic physical disability in children. It is attributed to a non-progressive disturbance of the developing fetal or infant brain. The brain lesion will cause primary problems, which include abnormal muscle tone, lack of strength, loss of selectivity and impaired balance. Secondary problems develop over time due to the combination of these primary problems and growth : short skeletal muscles, fixed contractures

- Lieven Vermuyten

- Katleen Desloovere ${ }^{1,2}$

- Guy Molenaers ${ }^{1,3}$

- Anja Van Campenhout ${ }^{1,3}$

${ }^{\prime}$ UZ Leuven Department of Orthopaedic surgery

${ }^{2}$ Laboratory for Clinical Motion Analysis (C-MAL)

${ }^{3} \mathrm{KU}$ Leuven Department of Development and Regeneration

Correspondence : Lieven Vermuyten, UZ Leuven Orthopedie, Herestraat 49, 3000 Leuven.Phone : 0163388 27, Fax : 016 338824.

Email : Lieven.Vermuyten@uzleuven.be

- 2021, Acta Orthopædica Belgica. 
and bony deformities. These result in progressive gait disturbances in ambulatory children (1).

A high femoral anteversion is one of the most frequent bony problems. It interferes with a functional gait as it often leads to internal hip rotation during stance phase leading to internal foot progression, tripping and falling. Further, biomechanical modelling shows that increased femoral anteversion in a $\mathrm{CP}$ child decreases the potential of specific muscles such as the hamstrings to generate hip and knee extension therefore increasing crouch gait (2). Additionally, increase in femoral anteversion results in increase of pelvic range of motion (3) Hence correction of the high femoral anteversion is often part of the treatment of gait problems in children with CP.

The treatment of this malrotation is surgical with femoral derotation osteotomy (FDO). FDO is often performed in the context of single event multiple surgery (SEMLS). SEMLS is a generally accepted surgical principle in ambulatory patients with CP in which all orthopedic problems, soft tissue and bony, are treated in one surgical session $(4,5)$. The indications for FDO, the amount of derotation needed, technique and outcomes have been discussed by a number of authors, giving some guidelines. (4-6) 3D gait analyses (3DGA) helps to define the indication to surgery and the amount of derotation needed. Functional outcome is superior if gait analysis, more specifically mean hip rotation in stance (MHR), is taken into account compared to clinical examination alone (6). Further it is an invaluable tool to assess functional outcome of SEMLS and FDO.

Still a substantial group of $\mathrm{CP}$ patients has variable outcomes after FDO, both at short term with under- and overcorrection of the hip rotation during gait but also at long term with recurrence of malrotation of the femur. (7-9).

So far there are few articles describing the effect of age at time of surgery on the outcomes of FDO on gait (9-12) and this with conflicting results. Some authors advocate age at surgery under 10 years as a possible risk factor for recurrence of internally rotated gait $(11,13,14)$. On the other hand, when a child walks with a severe internal femoral rotation, this will often cause or increase a flexed gait. The choice between doing the FDO early with the possible risk of recurrence of internal rotation versus doing the FDO late with risk of progression of the flexed gait to knee extension deficit and patella alta - leading to the need for more invasive surgery - remains a challenge.

To gain more insight in the risk of recurrence after FDO in children with $\mathrm{CP}$, the results of proximal FDO at short and long term follow up will be assessed in a retrospective study. Both outcomes of the effect of FDO on hiprotation in gait as on overall gait will be described. We hypothesize that there is no significant difference in over- and undercorrections and sustainment of the correction of internally rotated gait when the FDO is performed before or after the age of 10 years.

\section{METHODS}

Patients with spastic diplegic $\mathrm{CP}$ that underwent proximal FDO at the University Hospital XXX (covered for review) between 2002 and 2013 with a minimum follow up of 3 years were assessed for inclusion. Inclusion criteria were children aged under 18 years at time of surgery with spastic diplegia, GMFCS levels I-III, presenting with increased femoral anteversion which results in gaitabnormaliteis. This FDO could be part of a SEMLS, but no other femur osteotomies than the proximal FDO were performed.

Exclusion criteria included any previous orthopedic surgical treatment or Botulinum toxin A injections 6 months prior to surgery. Patients with an incomplete pre- or postoperative evaluation comprising clinical examination and 3D gait analysis were also excluded, as were patients that needed pelvic surgery for hip dysplasia.

Study was approved by the local ethical committee (S number :58023)

All patients underwent a full clinical lower limb assessment by senior trained physical therapists on the day of gait analysis, including passive ROM of the hip with rotation measured prone. Clinical midpoint is defined as the subject lay prone, the knee was flexed to $90^{\circ}$, and the limb was rotated until the position of maximum lateral trochanteric prominence was felt. Femoral anteversion was 
Table 1. - Patient characteristics

\begin{tabular}{|l|c|c|c|c|}
\hline & FU 0 & FU 1 & FU 3 & FU 5* \\
\hline Age (years) & $10.68 \pm 3.31$ & $11.83 \pm 1.53$ & $14.06 \pm 3.52$ & $15.39 \pm 3.08$ \\
\hline Mean FU (years) & & $1.05 \pm 0.18$ & $3.16 \pm 0.53$ & $5.02 \pm 0.49$ \\
\hline Number of patients/ limbs & $31 / 55$ & $31 / 55$ & $31 / 55$ & $22 / 38$ \\
\hline Unilateral/ Bilateral FDO & $7 / 24$ & $7 / 24$ & $7 / 24$ & $6 / 16$ \\
\hline Men/women & $20 / 11$ & $20 / 11$ & $20 / 11$ & $13 / 9$ \\
\hline GMFCS I/II/III & $12 / 10 / 9$ & & & \\
\hline
\end{tabular}

Table 2. - Results of clinical examination and instrumented 3D gait analysis

\begin{tabular}{|l|c|c|c|c|}
\hline Parameters & FU0 (Pre-op) & FU1 & FU3 & FU5 \\
\hline Clinical examination & $68.73 \pm 9.29$ & $41.18 \pm 11.4^{* *}$ & $48 \pm 11.21^{\Delta \Delta}$ & $48.95 \pm 12.03$ \\
\hline Passive internal hip rotation & $27.18 \pm 9.22$ & $30.63 \pm 11.22$ & $31.18 \pm 11.97$ & $27.89 \pm 10.04$ \\
\hline Passive external hip rotation & $47.95 \pm 5.23$ & $35.90 \pm 8.665^{* *}$ & $39.59 \pm 8.19^{\Delta}$ & $38.42 \pm 7.91$ \\
\hline Clinical midpoint & $27.55 \pm 14.3$ & \\
Amount of derotation & $36.27 \pm 6.86$ & $20.57 \pm 7.49^{* *}$ & $20.09 \pm 7.23$ & $20.80 \pm 6.42$ \\
\hline Anteversion & $-2.55 \pm 6.86$ & $1.18 \pm 3.96^{*}$ & $2.18 \pm 4.88$ & $2.63 \pm 7.60$ \\
Knee extension & $-1.34 \pm 8.36$ & $-0.55 \pm 8.02$ & $-032 \pm 6.86$ & $-0.78 \pm 6.57$ \\
\hline Kinematics & $11.44 \pm 16.09$ & $1.81 \pm 11.96^{* *}$ & $2.94 \pm 9.19$ & $5.11 \pm 9.08$ \\
\hline Mean pelvic rotation in stance & $27 \pm 9.90$ & $32.66 \pm 10.51^{*}$ & $30.05 \pm 8.31^{\Delta}$ & $31.33 \pm 10.01^{\S}$ \\
\hline Mean hip rotation in stance & $4.13 \pm 16.56$ & $-4.21 \pm 17.05^{* *}$ & $-5.34 \pm 9.56$ & $-2.42 \pm 9.634$ \\
\hline Max knee extension in stance & $15.65 \pm 8.51$ & $12.15 \pm 6.14^{* *}$ & $10.49 \pm 2.93$ & $11.19 \pm 4.48$ \\
\hline Mean foot progression angle & $17.09 \pm 8.90$ & $12.13 \pm 6.37^{* *}$ & $12.9 \pm 5.67$ & $13.17 \pm 5.87$ \\
GPS & $20.7 \pm 8.48$ & $17.29 \pm 10.47^{* *}$ & $15.4 \pm 6.44$ & $15.17 \pm 6.20$ \\
MAPS Hip sag & \multicolumn{3}{|}{} \\
MAPS Knee sag &
\end{tabular}

examined using the trochanteric prominence angle test as described by Ruwe et al. (15).

Lower limb dimensions and body height were measured to enable an estimation of joint centres. Skin mounted markers were applied following the protocol of Kabada et al (16). Subjects walked barefoot on a $10 \mathrm{~m}$ walkway at self-selected comfortable speed. Kinematic measurements were collected using a VICON system with 8 infrared cameras (Nexus capturing system measuring at $100 \mathrm{~Hz}$, with lower limb Plug-In-Gait marker set, VICON, Oxford Metrics, Oxford, UK) and two AMTI force plates (Advanced Mechanical Technology, Inc., Watertown, MA, USA). At least three valid barefoot walking trials were collected. After processing the files in VICONTM, extraction of data was done with Mathlab ${ }^{\mathrm{TM}}$ (Mathworks, Massachusetts, U.S.A). Each patient's results for all three trials were averaged before further analysis with SPSS (IBM, New York, U.S.A.).
All surgeries were performed by one of two senior orthopedic surgeons.

Both the amount of passive internal and external rotation and the clinically assessed femoral anteversion (15) were combined with MHR in stance and pelvic anterior tilt during gait analysis to define the amount of derotation needed. Patients were operated in prone position to obtain a good assessment of the amount of correction of rotation. FDO was performed using a lateral approach, intertrochanteric osteotomy and fixation with cannulated pediatric osteotomy system CAPOS (DePuy-Synthes, Switserland). Postoperative management included early mobilization with weight bearing transfers after 3-4 weeks and subsequent ambulation.

Gait analysis and clinical exam was performed at baseline (maximum 6 months before FDO), one and three years after FDO and if present at maximal further follow-up. Operated limbs are analyzed instead of patient. This was done to prevent missing 
under- and overcorrection by taking the average of two operated limbs. Furthermore, analyses were made on the whole subset of values instead of their averages, again to avoid masking under- and overcorrections (7).

Primary outcome measure was MHR during stance phase. Further a set of clinical parameters (hip rotation and anteversion) and gait parameters (table 2) were selected. Gait Profile Score (GPS) was also assessed as overall measure of the quality of gait (17).

Comparison of outcome parameters at the different time points was done using the Wilcoxon test because of small sample size and a nonnormal data distribution. To statistically analyse overall changes during follow up a related sample Friedman's two-way analysis of variance (ANOVA) was performed.

A second analyses with age-based groups (age at time of surgery $<10 \mathrm{y}$ or $>10 \mathrm{y}$ ) used a MannWhitney test because the samples were non-paired.

An analysis of preoperative average MHR between age groups was performed with a two tailed $\mathrm{T}$ test with equal variance.

A statistical significance of $p<0.05$ was used for all performed statistics.

\section{RESULTS}

After applying in- and exclusion criteria on an initial database of $318 \mathrm{CP}$ patients that underwent proximal FDO, 55 patients were eligible for inclusion (table 1). Insufficient or incomplete data for the given follow up points caused an exclusion of a further 24 patients bringing the end total to 31 patients (55 operated limbs); table 1. This group consisted of 20 boys and 11 girls, aged on average 11years $(10.68 \pm 3.31)$ at the time of surgery. Minimum follow up was 3 years $(3.16 \pm 0.53)$, with 22 patients ( 38 operated limbs) having an additional follow up at 5 years $(5.02 \pm 0.49)$. Mean time from preoperative analysis until surgery was 5 months $(0.38 \pm 0.27$ years $)$.

Before surgery, the average amount of hip internal rotation was $69^{\circ}\left(68.72 \pm 9.29^{\circ}\right)$, mean external hip rotation was $27^{\circ}\left(27.18 \pm 9.22^{\circ}\right)$ and femoral anteversion $36^{\circ}\left(36.27 \pm 6.86^{\circ}\right)$. The mean amount of derotation performed was $28^{\circ}\left(27.55 \pm 14.3^{\circ}\right)$.

Average correction of the MHR in stance from $11.44 \pm 16.09^{\circ}$ to an almost neutral rotation $\left(1.81 \pm 11.96^{\circ}\right)$, although with wide variation, was observed after one-year follow-up.

Further, a significant decrease in passive internal hip rotation and clinical midpoint were recorded.

Clinically measured femoral anteversion was corrected from an average of about $36^{\circ}$ to $20^{\circ}$. GPS dropped from an average of $15.65^{\circ}$ to $12.15^{\circ}$.

There was no change in mean pelvic rotation in stance phase.

At 3- and 5-year follow-up, the MHR in stance didn't change significantly, but had a tendency to mild deterioration $\left(2.94^{\circ}\right.$ at $3 \mathrm{y}$ and $5.11^{\circ}$ at $\left.5 \mathrm{y}\right)$ although with less variation.

Passive internal hip rotation and clinical midpoint did significantly increase again but remained far less than preoperatively. The clinically assessed femoral anteversion remained stable. GPS further
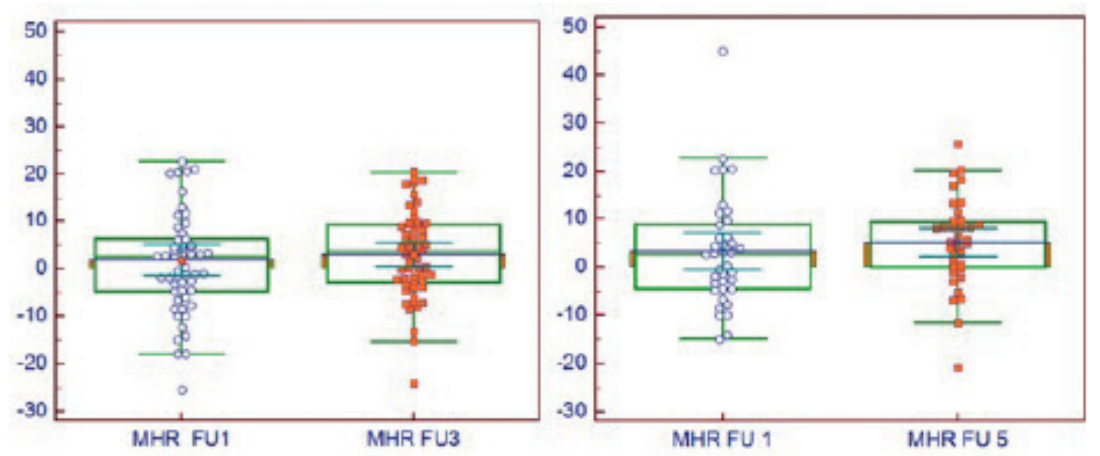

Fig. 1. - Box plot showing no significant difference in MHR at FU3 compared to FU1 (left) or FU5 compared to FU1 (right) Analysis performed with Wilcoxon test with significance level set at $\mathrm{p}<0.05$. $\mathrm{P}$ values are 0.14 and 0.35 . 

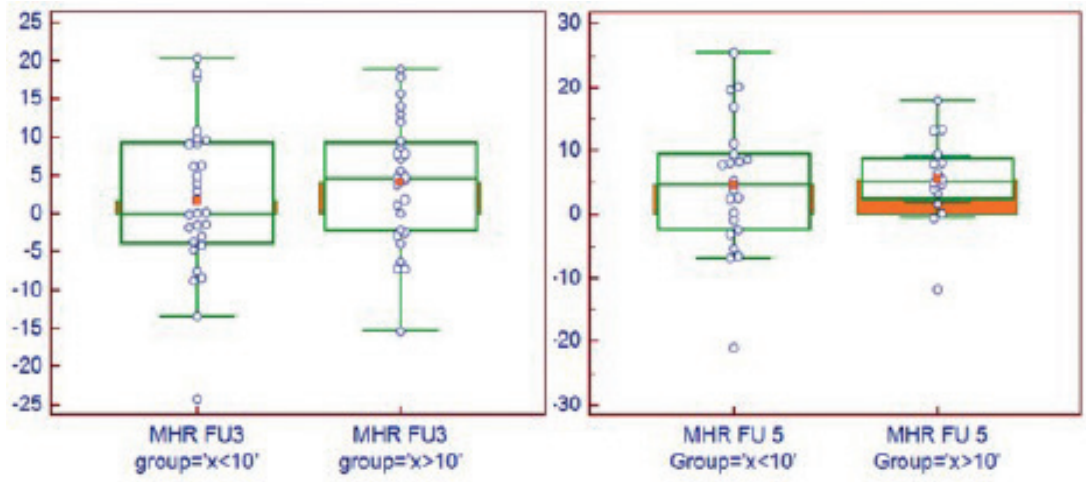

Fig. 2. - Box plot showing no significant difference in MHR at FU3 (left) or FU5 (right) with age groups. Cut off of 10 years was used. Analysis performed with Mann-Whitney test with significance level set at $p<0.05$. $p$ values $p=0.0346$ fir FU3 and $p=0.745$ for FU5.

improved although not statistically significant to an average of 10.49 at 3 years and remained stable at 11.19 at 5 years follow-up. MAPS for hip and knee in sagittal maintained the correction achieved at 1 -year follow-up.

We defined a good correction as a postoperative MHR between $-15^{\circ}$ and $15^{\circ}(8)$. Out of 55 limbs, 46 $(83.6 \%)$ demonstrated a good correction after 1-year follow-up ; 6 limbs (10.9\%) were undercorrected $\left(\mathrm{MHR}>15^{\circ}\right)$ with an average MHR of $23.77^{\circ} \pm 9.58^{\circ}$. 3 limbs (5.5\%) were overcorrected $\left(\mathrm{MHR}<-15^{\circ}\right)$ with an average MHR of $-20.44 \pm 4.26^{\circ}$.

At 3 years follow-up, out of 55 limbs 2 limbs went from overcorrected to well corrected and 5 well corrected limbs $(9.09 \%)$ showed regression to internal rotation (with mean $17.80^{\circ} \pm 1.21^{\circ}$ ).

37 out of $42(88 \%)$ patients with 5 years followup had a satisfactory MHR, with the 5 unsatisfactory MHR being overcorrections (mean $20^{\circ} \pm 3.3^{\circ}$ ).

Paired analysis between groups of patients older versus younger than 10 years at time of surgery $(n=$ 28 and $n=27$ resp. for FU3, $n=22$ and $n=16$ resp. for FU5) found no difference for average MHR preoperatively and at 3- and 5-years follow-up (Figure 2).

The 5 operated limbs that showed recurrence after 3 years after initial correction at 1 year had a mean age of $11.04 \pm 4.6$ years and those who retained correction $(n=39)$ had a mean age of $10.21 \pm 3.17$ years); this difference was not statistically significant.

\section{DISCUSSION}

This retrospective study on the outcome of proximal FDO in 31 spastic diplegic $\mathrm{CP}$ children (55 limbs) demonstrated that $83.6 \%$ of limbs after 1 year and $88 \%$ after 5 years showed a good correction of MHR. Average MHR showed a slight but not statistically significant loss of correction after 3 and 5 years.

Clinically assessed femoral anteversion remained corrected to the same degree as 1 year postoperatively, but the amount of passive internal rotation increased at 3 years follow-up.

Our retrospective analysis of preoperative passive hip internal rotation and amount of peroperative derotation shows our indication and amount of derotation to be similar to other authors $(7,12,18)$. The amount of under- and overcorrection totalled 9 limbs (16.3\%). The amount of recurrence at FU3 $(9.09 \%)$ does not seem to differ largely from other authors results of $0 \%$ (12), 10\% (14), 15\% (19) and $33 \%$ (13).

One of the advantages of our study is the implementation of the GPS. In our study group, GPS dropped from an average of $15.65^{\circ}$ to $12.15^{\circ}$, which underscores a clinically significant improvement of overall gait.

Mean pelvic rotation did not seem to be affected significantly, nor did it show any significant difference between age dependent groups, so the role of pelvic rotation in our cohort remains unclear. 
Although pelvic retraction was uttered to be an dynamic compensatory mechanism for internally rotated gait (20), no changes were noted after FDO.

The average age after 5 years follow-up of 15.4 years means that most of our patients were past their growth spurt, meaning the beneficial effects of surgery sustained the negative impact of growth on gait parameters (1). This finding further backs findings reported by Dreher et al. (19).

Operation at a younger age, this is younger than 10 years, does not predispose to a higher risk for recurrence to internal rotated gait after FDO in our study. This was demonstrated when comparing parameters age-grouped and when analysing age as a factor within the recurrence group plotted versus the sustaining correction group.

Although difficult to generalize our findings, they would suggest no clear contra-indication on operating on younger patients and that the general trend should be to aim for a full correction or even slight overcorrection of MHR to allow for a mild recurrence of internal rotated gait and MHR, allthough this was not significant in our group.

Niklasch et al (12) found more recurrence in younger children in a study on 27 patients assessing both proximal and distal FDO. Of note is that hs study started with 70 patients but only retaining those with good correction after 1 year.

Kim et al (13) also reported more recurrence in younger children after distal FDO in 30 patients (45 limbs) with a follow-up of 6.5 years; de Morais Filho et al (14) had the same conclusion for proximal FDO with a recurrence rate of $33 \%$ in 35 patients after 53 months. Ounpuu et al (5) and Braatz et al (11) did not find a higher risk for recurrence at young age.

Limitations to our study are its retrospective design and lack of a control group. More importantly, the current cohort of patients studied might not be big enough to reveal underlying differences. When comparing the number of patients of Braatz et. (10) al and Ounpou et al. (12) they also had smaller groups of 45 and 20 patients respectively. This compared to the patient groups of De Morais et al. (14), Kim et al. (13) and Niklasch et al. (11) with respectively 35,30 and 27 patients at final follow-up. The fact that our subgroup of patients with 5-year follow- up probably are not all mature might mean that an underlying age dependent difference at longer follow up is missed. A longer follow-up and larger groups are needed.

On the other hand, this is the first study on the outcome of a substantial group of spastic diplegic children with proximal FDO that reports both details of correction of rotation and correction of overall gait profile on short- and long-term follow-up.

\section{CONCLUSION}

This study shows significant correction of internally rotated gait and overall gait profile after proximal FDO as part of SEMLS in our overall patient group. These changes are maintained at 3 and 5 years postoperatively.

There was no correlation between age and recurrence of internal hip rotation nor was age a significant variable in a sustainment of correction group versus a recurrence group plotted analysis. This implies there is no clear contra-indication on operating on younger patients.

Our findings suggest that the general trend should be to aim for a full correction or even slight overcorrection of MHR to allow for a mild recurrence of internal rotated gait and MHR.

Further analysis on large groups with longer follow up is needed to further outline the optimal and minimal age of proximal FDO.

\section{REFERENCES}

1. Bell KJ, Ounpuu S, DeLuca PA, Romness MJ. Natural progression of gait in children with cerebral palsy. $J$. Pediatr. Orthop. 2002 ; 22(5) : 677-82.

2. Bosmans L JK, Wesseling M, Molenaers G, Scheys L, Jonkers I. The role of altered proximal femoral geometry in impaired pelvis stability and hip control during CP gait : A simulation study. Gait \& posture. 2016 ; 44(2) : 61-7.

3. Radler C KA, Manner HM, Höglinger M, Ganger R, Grill F. Torsional profile versus gait analysis : consistency between the anatomic torsion and the resulting gait pattern in patients with rotational malalignment of the lower extremity. Gait \& posture. 2010 ; 32(3) : 405-10.

4. Thomason P, Selber P, Graham HK. Single Event Multilevel Surgery in children with bilateral spastic cerebral palsy : a 5 year prospective cohort study. Gait \& posture. $2013 ; 37(1): 23-8$. 
5. Rodda JM, Graham HK, Nattrass GR, Galea MP, Baker R, Wolfe R. Correction of severe crouch gait in patients with spastic diplegia with use of multilevel orthopaedic surgery. The Journal of bone and joint surgery American. 2006 ; 88(12) : 2653-64.

6. Filho MC, Yoshida R, Carvalho Wda S, Stein HE, Novo NF. Are the recommendations from three-dimensional gait analysis associated with better postoperative outcomes in patients with cerebral palsy? Gait \& posture. $2008 ; 28(2)$ : 316-22.

7. Dreher T, Wolf S, Braatz F, Patikas D, Doderlein L. Internal rotation gait in spastic diplegia - critical considerations for the femoral derotation osteotomy. Gait \& posture. $2007 ; 26(1): 25-31$.

8. Niklasch M, Dreher T, Doderlein L, Wolf SI, Ziegler $\mathbf{K}$, Brunner R, et al. Superior functional outcome after femoral derotation osteotomy according to gait analysis in cerebral palsy. Gait \& posture. 2015 ; 41(1) : 52-6.

9. Schwartz MH, Rozumalski A, Novacheck TF. Femoral derotational osteotomy : surgical indications and outcomes in children with cerebral palsy. Gait \& posture. 2014 ; 39(2) : 778-83.

10. Braatz F, Poljuchow J, Klotz MC, Heitzmann DW, Wolf SI, Dreher T. Femoral Derotation in Children with Cerebral Palsy - Does the Result Depend on the Age at Operation and the Kind of Surgery? Zeitschrift fur Orthopadie und Unfallchirurgie. 2015.

11. Niklasch M, Wolf SI, Klotz MC, Geisbusch A, Brunner $\mathbf{R}$, Doderlein L, et $\boldsymbol{a l}$. Factors associated with recurrence after femoral derotation osteotomy in cerebral palsy. Gait \& posture. $2015 ; 42(4): 460-5$.

12. Ounpuu S, DeLuca P Fau - Davis R, Davis R Fau Romness M, Romness M. Long-term effects of femoral derotation osteotomies : an evaluation using three-dimensional gait analysis. 2002 (0271-6798 (Print)).

13. Hayong Kim M, Michael Aiona, Michael Sussman. Recurrence after femoral derotational osteotomy in cerebral palsy. 2005.

14. de Morais Filho MC, Kawamura CM, dos Santos CA, Mattar R, Jr. Outcomes of correction of internal hip rotation in patients with spastic cerebral palsy using proximal femoral osteotomy. Gait \& posture. $2012 ; 36(2)$ : 201-4.

15. Ruwe PA, Gage JR, Ozonoff MB, DeLuca PA. Clinical determination of femoral anteversion. A comparison with established techniques. The Journal of bone and joint surgery American. 1992 ; 74(6) : 820-30.

16. Kadaba MP, Ramakrishnan HK, Wootten ME. Measurement of lower extremity kinematics during level walking. $J$. Orthop. Res. $1990 ; 8(3)$ : 383-92.

17. Baker R, McGinley JL, Schwartz MH, Beynon S, Rozumalski A, Graham HK, et al. The Gait Profile Score and Movement Analysis Profile. Gait \& posture. 2009 ; 30(3) : 265-9.

18. Pirpiris M, Trivett A, Baker R, Rodda J, Nattrass GR, Graham HK. Femoral derotation osteotomy in spastic diplegia : Proximal or distal? The Journal of Bone and Joint Surgery. $2003 ; 85(2): 265-72$.

19. Dreher T, Wolf SI, Heitzmann D, Swartman B, Schuster W, Gantz S, et al. Long-term outcome of femoral derotation osteotomy in children with spastic diplegia. Gait \& posture. $2012 ; 36(3)$ : 467-70.

20. Kay RM, Rethlefsen S, Reed M, Do KP, Skaggs DL, Wren TA. Changes in pelvic rotation after soft tissue and bony surgery in ambulatory children with cerebral palsy. $J$. Pediatr. Orthop. 2004 ; 24(3) : 278-82. 